\title{
Подход к проектированию программного обеспечения систем управления искусственнъми сущностями
}

Г.П. Виноградов 1,2, д.т.н., профессор, заведующий лабораторией, wgp272ng@таil.ru И.А. Конюхов 2, заведуюший отделом, konuhov@сps.tver.ru

Г.А. Шепелев ${ }^{2}$, инженер-программист

${ }_{1}^{1}$ Тверской государственный технический университет, г. Тверь, 170026, Россия ${ }^{2}$ НИИ "Центрпрограмисистем", г. Тверь, 170024, Россия

Задачи интеллектуального управления искусственными сущностями (в том числе роботизированными комплексами) тесно связаны с проблемой принятия решений. Формальная теория выбора развивалась путем абстрагирования от субъективных факторов. Это привело к созданию нормативной теории принятия решений «идеальным» субъектом. Анализ подходов к построению систем управления роботизированными комплексами показал, что они не обладают способностью самостоятельно принимать решения. На практике разработчики продумывают возможные варианты поведения таких систем, и соответствующие алгоритмы закладываются в систему управления роботизированными комплексами. Как результат - такой объект не обладает свойством самодостаточного поведения, гарантирующего выполнение некоторой миссии, особенно в составе человеко-машинной системы. Требование интеллектуализации поведения заставляет пересмотреть логические и математические абстракции, положенные в основу построения их бортовых систем управления.

Цель работы - обосновать подход к разработке ПО интеллектуальных систем управления роботизированными комплексами на базе теории паттернов. Разработать подход, обеспечивающий перенос эффективного опыта в систему управления роботизированными комплексами, совместимость теологического подхода и подхода, основанного на причинно-следственных связях, что важно при интеграции роботизированных комплексов и личного состава подразделений, показать, что закономерности отхода субъекта от идеального рационального выбора к субъективно рациональному связаны с особенностями идентификации и понимания состояния внешнего окружения и свойств своих интересов. Внешние факторы связаны с обязательствами, которые принимает на себя агент. Внутренние факторы отражают интересы субъекта, индуцируемые его потребностями и этической системой, которой он придерживается.

В работе использованы методы теории рефлексивных игр и теории информационного управления системами, обладающими волей и интеллектом.

Показано, что выбор в условиях жесткого дефицита времени осуществляется на основе паттернов поведения, отражающих эффективный опыт. Паттерны образуют как информационную структуру представлений, так и множество возможных вариантов представлений. Оценки удовлетворенности текущей ситуацией выбора субъектом приводят к изменению структуры интересов субъекта, и он может ее выбирать. Разработана формальная модель паттерна поведения. Предложен подход к решению проблем идентификации и построения моделей паттернов, используя для этого четыре позиции обработки информации. Разработан метод логического вывода на паттернах. Приведены результаты программных решений идентификации паттерна поведения при использовании тренажерных систем нового поколения.

Ключевые слова: принятие решений, иелеустремленные системы, нечеткое суждение, паттерн, ситуация выбора, РТК.

В настоящее время активно идет процесс интеграции роботизированных комплексов (РТК) и личного состава войсковых соединений. Такой новый объект должен обладать самодостаточным поведением, гарантирующим выполнение некоторой миссии. Реализовать эту способность возможно, главным образом, путем направления усилий конструкторов и ученых на совершенствование интеллектуальной составляющей их системы управления, а именно: совокупности алгоритмов бортовых систем управления РТК и алгоритмов деятельности личного состава, осуществляющего управление РТК. Эти компоненты образуют кооперативный интеллект РТК, который позволяет из набора разрозненных систем бортового 
оборудования создать функционально целостный объект, нацеленный на выполнение задачи текущего сеанса функционирования соединения.

Автономный интеллектуальный РТК (будем называть его агентом), проявляющий поведение, подобное человеческому, - это система, имеющая в своем составе следующее:

- бортовые измерительные устройства (или комплекс бортовых измерительных устройств), выполняющие роль сенсоров, позволяющих получить информацию о состоянии внешней среды и собственном состоянии;

- бортовые исполнительные устройства (или комплекс бортовых исполнительных устройств), с помощью которых система воздействует на внешнюю среду и на саму себя, выполняющие роль эффекторов;

- средства коммуникации с другими системами;

- бортовой интеллект, составляющими которого могут быть бортовые вычислительные машины, их ПО, а также операторы пункта управления, являющиеся носителями набора алгоритмов для решения задач предметной области, полученный за счет обучения, тренировок и накопления опыта.

Такая система существует во времени и пространстве, взаимодействует с другими агентами и со средой при выполнении боевых задач с помощью доступных ему способов действия. Агент выполняет поставленные задачи исходя из понимания своего состояния и субъективных представлений о состоянии среды и развитии боевой ситуации на основе информации, полученной через модуль коммуникации. Агент должен быть способен прогнозировать изменения среды от своих действий и оценивать их полезность.

Управление эффективной и согласованной работой всех подсистем интеллектуальной автономной искусственной сущности имеет информационный характер. Однако в связи с его реализацией возникают вопросы:

- создания информационных процессов, обеспечивающих работу управления, подобного человеческому;

- создания моделей и алгоритмов познания процессов и явлений предметной области, в которой будет существовать искусственная сущность;

- использования результата познания для реализации успешного поведения искусственной сущности;
- создания алгоритмов и моделей преобразования познавательных способностей в интеллект.

Решить их можно, разработав искусственные сущности, использующие символьные или нейросетевые методы и модели приобретения и формализации знаний [1]. Онтологии сделали методы искуственного интеллекта (ИИ) и модели знаний интерпретируемыми в естественно-языковой семантике и вывели проблему человеко-машинного взаимодействия на новый уровень [2, 3].

Однако следует отметить, что подавляющее число исследований в этой области остаются на теоретическом уровне [4-8]. Существует разрыв между примитивными моделями поведения искусственных сущностей, например, в роевой робототехнике, моделями их взаимодействия и ожиданиями со стороны практики [9-11].

\section{Требования к автономии и интеллектуальности боевых автономных систем}

Роль автоматизированных систем при выполнении боевых задач следует рассматривать с позиции их влияния на человека. Они должны помогать командиру, упрощая и повышая эффективность его работы. Командир при этом обязан быть элементом системы управления РТК. Их взаимодействие должно сделать возможной передачу опыта как от человека к машине, так и в обратном направлении, обеспечивая тем самым приспособительное поведение. Например, главной сложностью для любой автономной системы является распознавание ситуаций в окружающей среде. Сложность и многочисленность ситуаций, возникающих при выполнении миссии, делают невыполнимыми задачу их выявления по результатам множественных испытаний и формирование на их основе базы знаний. Следовательно, необходимо реализовать дополнительную схему мониторинга роботизированной системы, чтобы выявить классы ситуаций и успешных способов действия для формирования моделей поведения (паттернов) на основе данных, получаемых в реальных условиях [12, 13]. Данная схема гарантирует управляемую эволюцию самодостаточности при решении задач боевыми подразделениями, имеющими в своем составе автономные РТК. При этом возникает следующая проблема: логические процессы происходят в человеческом мышлении, процессы, которые 
человек познает посредством логики, относятся к изучаемой человеком предметной области. Возникает вопрос о возможности использовать процессы первого типа для изучения процессов второго типа.

\section{Исходные предположения и гипотезы}

Одно из правил логического вывода - правило modus ponens - гласит: если имеет место А и из А следует В, то имеет место В, или $(\mathrm{A}, \mathrm{A} \rightarrow \mathrm{B}) \Rightarrow \mathrm{B}$. Необходимо выяснить, как человек, наблюдая, что за явлением А постоянно следует явление В, приходит к выводу, что А является причиной В, а также то, какой механизм сознания позволяет определить, что А есть причина В.

Будем считать, что этот механизм является встроенным, назовем его паттерном познания (моделью или алгоритмом познания). Кант называл его «системой чистого разума» и показал, что она имеет априорный характер: то есть он (паттерн познания) существует в человеческом сознании прежде всякого опыта и является основой и полученного знания относительно процессов, и явлений предметной области.

Поэтому, пока отсутствует формальная модель паттерна познания, наиболее конструктивным и эффективным при моделировании адаптивного поведения следует считать феноменологический подход. Согласно ему, существуют формальные правила интеллектуального поведения, и эти правила не обязательно связаны с конкретными нейронными структурами, имеющимися у живых организмов.

Как правило, ситуации, возникающие перед автономной системой, достаточно сложны для конструктивной формализации их традиционными формальными методами, но они хорошо описываются средствами естественного языка и имеется опыт их лучшего разрешения и описания в естественно-языковой семантике. Носитель такого опыта называется лидером. Опыт лидеров передается с помощью средств коммуникации на выбранном языке, что обеспечивает его переносимость. Это первая гипотеза.

Вторая гипотеза: переживания/поведение человека следует рассматривать как функцию взаимодействия ситуации и человека. Ситуацию можно интерпретировать как компонент причины, которая порождает субъективное отражение ее у человека. Человек, выбирая определенное поведение на основе субъективного представления ситуации, оказывает влияние на ситуацию, изменяя ее. В то же время процессы, происходящие в сознании человека при выполнении определенных действий, приводят к расширению его структуры способностей (знания, опыт). Таким образом, деятельность надо рассматривать как последовательность ситуаций, определяемую целеустремленным выбором. Модель поведения РТК также должна учитывать этот феномен взаимовлияния.

При таком подходе конструктивным оказалось понятие типовая ситуация (ТС) [14, 15]. Это функционально замкнутая, с четко обозначенной значимой целью часть работы РТК, которая как единое целое встречается в различных (реальных) сеансах, конкретизируясь в них по условиям протекания и по доступным способам разрешения возникающих в ТС проблемных субситуаций [9]. ТС и способы действия как реакция на нее образуют индивидуальный паттерн поведения. Структура ТC на данном уровне обобщения сохраняется, меняются только значения показателей (переменных ситуации). Новая ТС имеет иную структуру. Человек нацелен на агрегирование приобретаемого опыта, создавая модели паттернов. Следовательно, модель паттерна необходимо рассматривать как единицу человеческого опыта, для которой в ситуации, схожей с типовой (кластер), у человека сформирована определенная степень уверенности в получении желаемых состояний. Автор работы [16] показал, что идеальная интеллектуальная система должна иметь 13 видов способностей. На современном этапе лишь часть из них можно реализовать, причем только в интерактивном режиме во взаимодействии с человеком. Например, «это порождение последовательности «цель-план-действие», способность к рефлексии, способность к интеграции знаний, способность к уточнению неясных идей, способность к изменению системы знаний при получении новых знаний». Он отмечает, что исключить человека из этого режима нельзя при отсутствии модели механизма познания. Поэтому интеллектуальная система для военных целей не может быть полностью автономной и должна рассматриваться как партнерская человеко-машинная система, единицей знаний которой должен быть паттерн.

\section{Теория паттернов}

Как установлено психологами, человек не контролирует свои действия и реакции ежесекундно, иначе ему пришлось бы постоянно 
задумываться над каждым словом и движением, сознание быстро уставало бы от таких объемов работы. Мозг следует более рациональной схеме: использовать готовую эффективную модель поведения, а не заново конструировать ответ в определенной ситуации. Естественно использовать такую же схему при управлении РТК для экономии вычислительных ресурсов.

Паттерн - это результат активности естественной или искусственной сущности, связанный с действием, принятием решений, его реализацией и т.п., осуществленный в прошлом и рассматриваемый как шаблон (образец) для повторных действий или как обоснование действий по этому шаблону. (Слово «паттерн» переводится как «образец, шаблон, шаблонная модель».)

В теории паттернов используются объекты или паттерны четырех уровней. Первый уровень составляют объекты, называемые образующими. Они моделируют относительно простые объекты реального мира, обладающие связями, которые могут соединяться со связями других объектов. Из образующих паттернов путем соединения их связей конструируются регулярные конфигурации, относящиеся к объектам второго уровня. Объектами третьего уровня теории служат изображения, получаемые следующим образом: сходные между собой регулярные конфигурации объединяются в множество, на котором определяются классы эквивалентности. Отношение эквивалентности, определяющее класс эквивалентности на множестве регулярных конфигураций, и называется изображением. Четвертый уровень объектов составляют образы. Образом называется множество изображений, инвариантных относительно преобразований подобия. Если на некотором множестве регулярных конфигураций существует только одно изображение, то оно представляет собой единственный образ этого множества. Образующие, регулярные конфигурации, изображения и образы являются, соответственно, паттернами первого, второго, третьего и четвертого уровней. Формальные методы теории паттернов, применяемые на каждом из четырех уровней, отличаются высокой степенью гибкости и позволяют моделировать связи, соединения и преобразования подобия логических объектов реального мира. Задачи рассуждений мозг решает путем обработки образов на нейросетевых структу$\operatorname{pax}[17]$.

\section{Общая архитектура интеллектуальной системы управления}

Система управления автономным РТК имеет иерархическую структуру. Верхний уровень, реализуемый командиром, соответствует таким свойствам, как выживание, безопасность, выполнение обязательств согласно миссии, накопление и корректировка базы знаний в виде эффективных паттернов поведения. Объектом управления для нее является РТК, рассматриваемый как некоторая функциональная система, осуществляющая расчет текущих показателей удельной ценности по результатам и эффективности в момент $t$, расчет и реализацию способа действия (поведения) в момент $t$ согласно заданному паттерну поведения, мониторинг результатов реализации паттерна поведения.

Структурно систему можно представить в виде двух блоков (рис. 1): искусственная сущность (РТК) и оператор (командир).

Командир отрабатывает паттерны поведения для ТС при выполнении миссии. Искусственная сущность рассчитывает последовательности состояний $y(t)$, реализует задачи миссии и выполняет расчет оценки удельной эффективности отработки фактических ситуаций $V(t)$. Командир выполняет анализ выполнения миссии: $\mu A$ (миссия выполнена) $\geq \alpha_{v}^{*}$.

\section{Модель нечеткого описания паттерна поведения}

Поведение в ТС связано с выбором, который происходит в ситуации целеустремленного состояния [10]. Рассмотрим модель поведения в виде нечеткого описания модели ситуации выбора. Возможный вариант такой конструкции предложено строить путем «парадигмальной прививки» идей, транслируемых из других наук, например $[10,18]$. Целеустремленное состояние складывается из перечисленных далее компонент.

- Субъект, осуществляющий выбор (агент), $k \in K$.

- Окружение выбора $(S)$, под которым понимается множество элементов и их существенных свойств, изменение в любом из которых может стать причиной или продуцировать изменение состояния целеустремленного выбора. Часть этих элементов может не являться элементами системы и образует внешнюю среду для нее. Воздействие внешней среды 


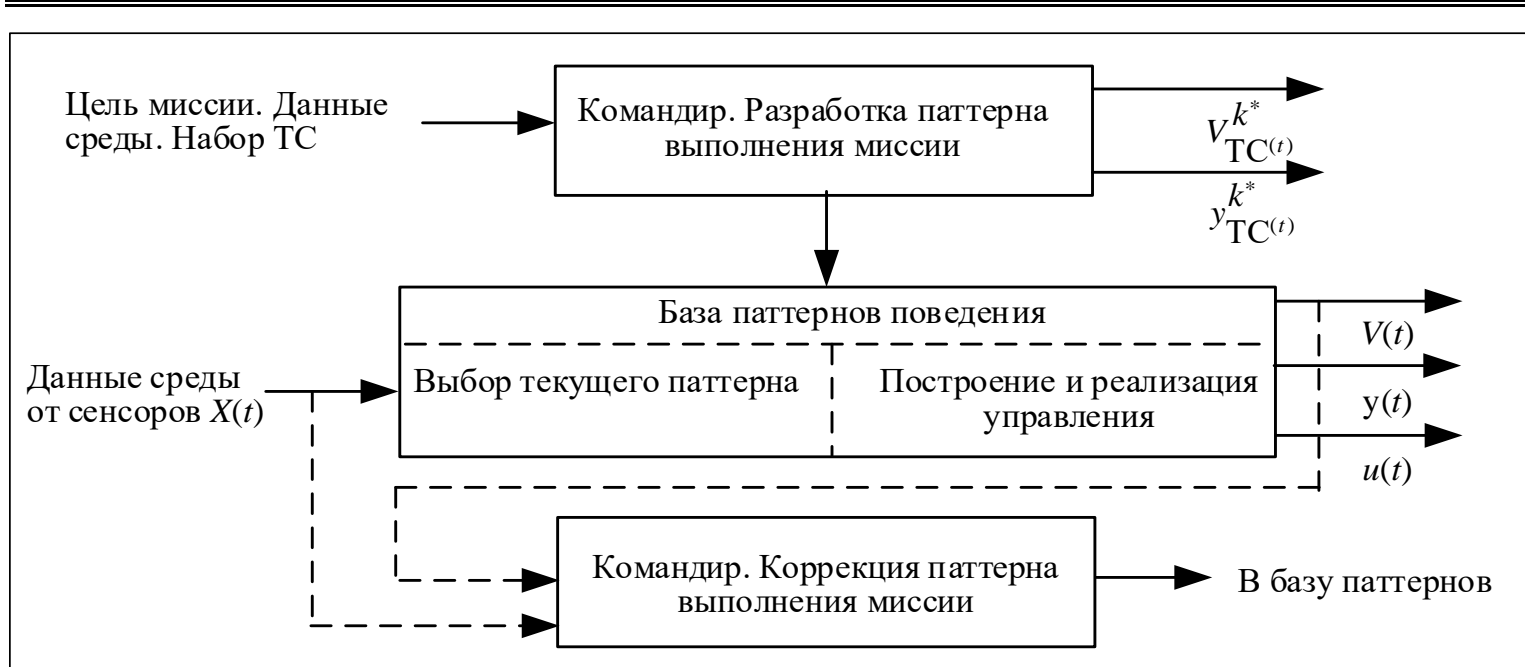

Рис. 1. Схема системы управления искусственной сущностью для двух тактов управления

Fig. 1. Control system configuration by an artificial entity for two control cycles

описывается с помощью некоторого набора переменных.

- Доступные способы действий $c_{j}^{k} \in C^{k}$, $j=\overline{1, n}, k$-го агента, которые ему известны и могут быть использованы для достижения $i$-го результата (их еще называют альтернативами). Каждый способ этого множества характеризуется набором параметров, называемых управляющими воздействиями.

- Возможные при окружении $S$ результаты, существенные для агента: $o_{i}^{k} \in O^{k}, i=\overline{1, m}$. Оценка результатов производится с помощью некоторого набора параметров, которые называются выходными параметрами ситуации целеустремленного состояния.

- Способ оценки свойств результатов, полученных вследствие выбора способа действия. Очевидно, что оценки результата должны отражать ценность результата для агента и тем самым отражать его индивидуальность.

- Ограничения, отражающие требования, накладываемые ситуацией выбора на выходные переменные и управляющие воздействия.

- Модель предметной области, которая представляет собой множество соотношений, описывающих зависимость управляющих воздействий, параметров и возмущений с выходными переменными.

- Модель ограничений агента. (Подробно описана в работе [15].) Независимо от используемого вида описания ограничений будем предполагать наличие у агента определенной степени уверенности в возможности изменения части ограничений в сторону расширения множества возможных вариантов (альтернатив) выбора.

Введем для описанных компонент меры, которые будут использоваться при оценке целеустремленного состояния.

1. Будем считать, что агент способен выделять факторы - характеристики окружения $X^{k}=\left\{x_{i}^{k}, i=\overline{1, N}\right\}$. Степень влияния каждого фактора агент оценивает с помощью лингвистической переменной $\mu_{x}^{k}\left(x_{i}^{k}\right): x_{i}^{k} \rightarrow[0,1]$.

Введем параметр для оценки агентом своей ситуационной осведомленности в ситуации целеустремленного состояния:

$$
E s^{k}=\frac{\sum_{i=1}^{N} \mu_{x}^{k}\left(x_{i}^{k}\right) x_{i}^{k}}{\sum_{i=1}^{N} \mu_{x}^{k}\left(x_{i}^{k}\right)} .
$$

Можно определить ограничение

$$
\sigma^{k}\left(E s^{k}\right) \geq \sigma_{0}^{k},
$$

где $\sigma_{0}^{k}-$ некоторый пороговый уровень осведомленности агента от использования собственных источников информации.

2. Будем предполагать, что для описания влияния выделенных факторов на результаты $o_{i}^{k}, i=\overline{1, m}$, агент использует аппроксимацию в виде продукционных правил:

если $x_{1}$ есть $A_{r 1}^{k}$ и если $x_{2}$ есть $A_{r 2}^{k}$ и ... и если $x_{N}$ есть $A_{r \mathrm{~N}}^{k}$, то $o_{i}^{k}=f_{i r}^{k}\left(x_{1}, x_{2}, \ldots, x_{N}\right)$, $r=\overline{1, R}, i=\overline{1, m}$

где $R$ - количество продукционных правил; $r-$ номер текущего продукционного правила; 
$o_{i}^{k}=f_{i r}^{k}\left(x_{1}, x_{2}, \ldots, x_{N}\right)$ - четкая функция, отражающая представление агента о причинно-следственной связи входных факторов с возможными результатами для $r$-го правила; $A_{r i}^{k}-$ нечеткие переменные, определенные на $X^{k}=\left\{x_{i}^{k}, i=\overline{1, N}\right\}$.

В качестве функции $f_{i r}^{k}(\bullet)$ могут использоваться, например, как математические модели, словесное описание, графики, таблицы, алгоритмы и т.д.

Поскольку $c_{j}^{k}$ является функцией параметров состояния внешней среды, принимаемых во внимание, свойств системы, то набор предположений об их возможных значениях образует сценарий возможного состояния внешней среды, функциональных возможностей системы. Реализация сценариев, например, с помощью правил (1) позволяет сформировать представление о возможных результатах $o_{i}^{k}$. Неравнозначность при выборе способа действия можно описать как степень уверенности в необходимости его применения для получения результата $o_{i}^{k}$. Эту оценку можно описать лингвистической переменной

$$
\psi_{j}^{k}=\psi_{j}^{k}\left(c_{j}^{k} \in C^{k} \mid s_{i} \in S \rightarrow o_{i}^{k}\right) \in[0,1] .
$$

Данная мера является индивидуальной характеристикой агента, которая может меняться в результате обучения и приобретения опыта, а также коммуникационного взаимодействия агентов между собой и с оператором. Поэтому $\psi_{j}^{k}=\psi_{j}^{k}\left(c_{j}^{k} \in C^{k} \mid s_{i} \in S, I^{k} \rightarrow o_{i}^{k}\right) \in[0,1]$, где $I^{k}-$ информация, которой располагает агент на момент времени $t^{k}$.

3. Выбор способа действий $c_{j}^{k}$ при принятии решения агентом в ситуации целеустремленного состояния для достижения результата $o_{i}^{k}$ связан, как показано в работе [10], с построением количественной оценки свойств выбираемого решения.

Список свойств и параметров формируется на основе опыта, знаний, интеллекта и глубины понимания им ситуации принятия решения. Правильное описание свойств и параметров способа действий - одно из основных условий того, что выбор $c_{j}^{k}$ приведет к достижению результата $o_{i}^{k}$. Выбор перечня свойств и характеризующих их параметров целиком зависит от агента (его индивидуальности).

Представим возможные результаты при заданном окружении выбора агента в виде $o_{i}^{k} \in\left\{o_{i j}^{k}, j=\overline{1, J}\right\}$, где $o_{i j}^{k}-$ множество возможных результатов при выборе $j$-го способа действия; $i \in I-$ множество результатов, принимаемых во внимание $k$-м агентом.

Очевидно, что $o_{i j}^{k}=o_{i j}^{k}\left(s_{i}\right), s_{i} \in S$.

4. Ценность результатов $o_{i}^{k}$. Поскольку $o_{i j}^{k}=o_{i j}^{k}\left(s_{i}\right)$, а $s_{i}=S\left(c_{j}^{k}\right)$, ценность $i$-го вида результата можно оценить лингвистической переменной $\varphi_{i}^{k}\left(o_{i}^{k}\left(c_{j}^{k}\right)\right) \in[0,1]$. Функция $\varphi_{i}^{k}\left(o_{i}^{k}\left(c_{j}^{k}\right)\right)$ для результата $o_{i}^{k}$ будет монотонным преобразованием, так как $\varphi_{i}^{k}(\bullet)$ переводит область значений функции $o_{i}^{k}\left(c_{j}^{k}\right)$ в множество значений лингвистической переменной. Так как базовому значению лингвистической переменной соответствуют нечеткие переменные, это преобразование переводит область значений функции $o_{i}^{k}$ в область значений базовых нечетких переменных.

5. Эффективность способа действия с точки зрения результата - это уверенность в получении данного результата этим способом действия при известных (или предполагаемых) затратах на его реализацию. Степень уверенности $E_{i j}^{k}$ в том, что некоторый способ действия $c_{j}^{k}$ будет приводить к результату $o_{i}^{k}$ в окружении $S$, если агент выберет именно его: $E_{i j}^{k}=E_{i j}^{k}\left(o_{i}^{k} \mid A\right.$ выберет $c_{j}^{k}$ в $\left.S\right) \in[0,1]$.

Она является лингвистической переменной и выражает индивидуальную оценку агентом последствий выбора с точки зрения затрат.

\section{Модель выбора агента при реализации паттерна}

Введенные три лингвистические переменные $\mu_{i}^{k}\left(x_{i}^{k}\right), \psi_{i j}^{k}, E_{i j}^{k}$ образуют модель представлений агента о ситуации целеустремленного выбора.

Поскольку $c_{j}^{k}$ можно описать в терминах $X_{i}^{k}$ и агент имеет представление о зависимости в виде базы правил, которая связывает $c_{j}^{k}$ и ценность возможного $i$-го результата $o_{i}^{k}$, можно определить ценность целеустремленного состояния по $i$-му результату $o_{i}^{k}$ для $k$-го агента в соответствии с правилом $[4,15]$ :

$$
E \varphi_{i}^{k}=\frac{\sum_{j \in J} \varphi_{i j}^{k}\left(o_{i j}^{k}\left(c_{j}^{k}\right)\right) \cdot o_{i j}^{k}\left(s^{k}\right)}{\sum_{j \in J} \varphi_{i j}^{k}\left(o_{i j}^{k}\left(c_{j}^{k}\right)\right)} .
$$


По аналогии можно определить ценность целеустремленного состояния для $k$-го агента по эффективности для $i$-го вида результата:

$$
E E_{i}^{k}=\frac{\sum_{j \in J} E E_{i j}^{k}\left(o_{i}^{k}\left(c_{j}^{k}\right)\right) \bullet \psi_{i}^{k}\left(c_{j}^{k}\right)}{\sum_{j \in J} \psi_{i}^{k}\left(c_{j}^{k}\right)} .
$$

Оценка агентом желательности целеустремленного состояния по $i$-му результату и эффективности его достижения в ситуации выбора задается как лингвистическая переменная [19] $\chi_{i 1}^{k}=\chi_{1}^{k}\left(E \varphi_{i}^{k}\right) \in[0,1], \quad \chi_{i 2}^{k}=\chi_{2}^{k}\left(E E_{i}^{k}\right) \in[0,1]$.

Можно определить следующие ограничения:

$$
\sum_{i} \chi_{i 1}^{k}\left(E \varphi_{i}^{k}\right) \geq \chi_{1}^{0} \quad \text { и } \sum_{i} \chi_{i 2}^{k}\left(E E_{i}^{k}\right) \geq \chi_{2}^{0},
$$

где $\chi_{1}^{0}$ и $\chi_{2}^{0}-$ ожидания агента от выполнения миссии, которые отражают баланс между затратами и достигнутыми результатами $o_{i}^{k}$.

Моделью ситуации выбора агента в ТС будем называть множество структурных и функциональных свойств, которыми, по его убеждению, обладает ситуация выбора и которые влияют на его удовлетворенность или неудовлетворенность ситуацией.

Есть еще одна группа факторов, определяющих реализацию результата: воля, склонность к рискам, самооценка, мотивированность. Эти факторы позволяют говорить о таком показателе, как уверенность $\rho_{i}^{k}\left(o_{i}^{k}\right)$ в получении результата $o_{i}^{k}$ в ситуации выбора при использовании одного из возможных способов действия: $c_{j}^{k} \in C^{k}$.

В соответствии с гипотезой о рациональном поведении агент формирует решение в соответствии с

$$
\begin{aligned}
& P_{i}^{k}(s \in S)=\underset{c_{j}^{k}}{\operatorname{Arg} \max }\left(\sum_{j \in J} E \varphi_{i}\left(o_{i}^{k}\left(c_{j}^{k}\right)\right)-E E_{i}^{k}\left(o_{i}^{k}\left(c_{j}^{k}\right)\right)\right) \\
& c_{j}^{k} \in C^{k}\left(I_{t}^{i}\right), I_{t}^{i} \subseteq M, o_{i}^{k} \in O^{k} \\
& \sum_{i} \chi_{i 1}^{k}\left(E \varphi_{i}^{k}\right) \geq \chi_{1}^{0}, \sum_{i} \chi_{i 2}^{k}\left(E E_{i}^{k}\right) \geq \chi_{2}^{0} \\
& \sigma^{k}\left(E s^{k}(X)\right) \geq \sigma_{0}^{k} .
\end{aligned}
$$

Поскольку выбор связан с представлениями агента о ситуации выбора, то в (2) необходимо включить базу знаний.

Соотношения (2) описывают паттерн поведения агента (киберфизической системы) при стремлении достичь $i$-го результата. Агент рассматривает этот паттерн как способ описания часто возникающей задачи, принцип и алгоритм ее решения, причем таким образом, что ее решение можно использовать много раз, ничего не изобретая заново.
Ценность целеустремленного состояния по результату $E \varphi_{i}^{k}$ и ценность целеустремленного состояния по эффективности $E E_{i}^{k}$ являются элементами интегрального показателя ценности целеустремленного состояния для $k$ го индивида $-\sum_{i} E \varphi_{i}^{k} \bullet E E_{i}^{k}$. Учитывая степень его уверенности в результате $\zeta_{i}^{k}$, получим показатель ожидаемой удельной ценности:

$$
E V_{k}=\frac{\sum_{i}\left(E \varphi_{i}^{k}-E E_{i}^{k}\right) \bullet \zeta_{i}^{k}}{\sum_{i} \zeta_{i}^{k}} .
$$

Таким образом, если два субъекта находятся в одной и той же ситуации выбора, то разница в их поведении должна проявляться в значениях оценок удельной ценности по результату и эффективности и в степени уверенности в достижении цели.

Соотношения $(2,3)$ означают, что для получения какого-либо результа агент располагает несколькими альтернативными способами достижения с разной эффективностью, и его уверенность в получении желаемого результата значительна. Такая модель индивидуального поведения автономного агента предполагает формирование базы знаний путем обучения на основе экспериментального опыта, что позволяет реализовать эволюцию «кооперативного интеллекта» благодаря использованию искусственного когнитивного процесса, аналогичного тому, что имеет место у естественных сущностей $[20,21]$. Отметим, что системы на основе знаний не имеют такой возможности, так как в них отсутствует компьютерная модель адаптивного поведения. Таким образом, общие принципы мышления агента являются вполне традиционными и включают три основные фазы (рис. 2):

- восприятие (получение данных и построение модели сцены в загруженном мире);

- познание (анализ и формирование сценария действий субъекта для достижения поставленных целей);

- исполнение намеченного сценария с постоянным сопоставлением ожидаемых и наблюдаемых результатов.

В отличие от других подобных систем в рассматриваемой системе реализация этих фаз осуществляется через два базовых механизма: абстрагирования и конкретизации, тесно связанных между собой.

Процесс получения знания в предлагаемой схеме аналогичен процессу биологической эволюции. Следовательно, критерием истинности 


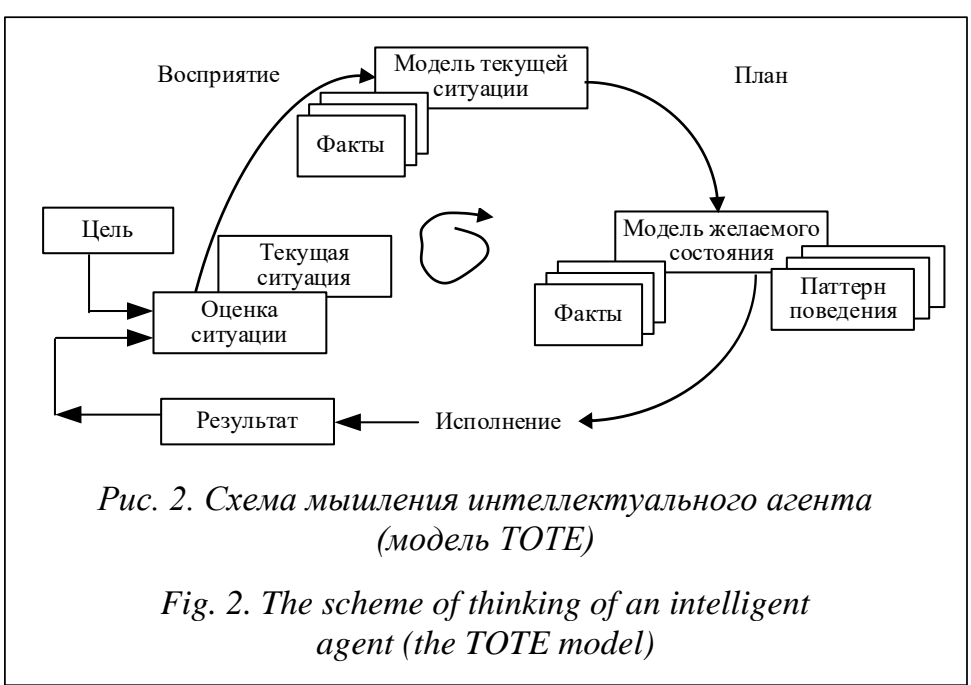

знания естественной или искусственной сущности является приспособленность его для решения задач предметной области.

Подход к моделированию поведения на основе паттернов аналогичен подходу в прагматической эпистемологии, где знания рассматриваются как множество теорий или моделей, каждая из которых представляет собой описание поведения некоторого класса явлений и предназначена для решения определенного класса задач. Теории, описывающие класс явлений, могут перекрываться, и возникает проблема выбора способа решения задач в конкретной предметной области. Критерием выбора становится ожидаемая удельная ценность возможного результата. Паттерны в этом случае дают корректное описание поведения агента в процессе выбора.

\section{Моделирование паттернов. Базовые позиции моделирования}

Моделирование паттернов выполняется с ограниченным подмножеством естественного языка, в том числе и моделирование рассуждений на паттернах, что образует специфическую часть человеческого опыта - метаопыт.

Для реализации описанного подхода разработана программная система, позволяющая производить моделирование среды (контекста) и паттерна поведения агента с различных позиций. Выделены четыре базовые позиции восприятия, с которых осуществляются сбор и интерпретация информации для идентификации модели паттерна поведения: 1) собственная точка зрения человека, 2) восприятие ситуации с точки зрения другого человека, 3) рассмотрение ситуации с точки зрения незаинтересованного наблюдателя, 4) рассмотрение ситуации с точки зрения системы, задействованной в ситуации.

Поскольку предполагается, что каждая позиция использует разные представления о ситуации и о возможных способах действия, интегрирование точек зрения и их согласование позволяют агенту расширить свое понимание ситуации целеустремленного состояния и паттерна поведения в ней.

Моделирование из первой позиции заключается в том, что человек, обладающий опытом выполнения миссии, реализует его в системе самостоятельно и исследует используемый при этом паттерн. Испытуемый реализует свое поведение, осуществляя голосовое управление аватаром в трехмерном пространстве (рис. 3).

Для экономии вычислительных ресурсов модель аватара представлена в виде композиции выделенных красными линиями прямоугольников. Недостаток этого способа в том, что снижается точность распознавания объекта, однако данный способ позволяет экономить аппаратные ресурсы и время на вычисление пересечений. Эта схема путем определения пересечений может использоваться, например, для реализации попадания в случае огневого контакта.

Основным элементом интерфейса в системе является микрофон. Данные, получаемые с него, проходят оцифровку и попадают в микроконтроллер, где происходят их нормировка и цифровая фильтрация (рис. 4). Затем в соответствии со звуковой командой формируется управляющий сигнал, который передается в компьютер через USB-интерфейс для манипулирования аватаром.

Сигнал, поступающий на микроконтроллер, содержит шумы, производимые изменением тембра голоса. Для удаления шумов из голосового сигнала решается задача линейной и нелинейной фильтрации управляющего сигнала по Калману [22-24].

Полученный управляющий код используется для вызова соответствующей процедуры для изменения поведения аватара. Для формирования сложного поведения аватар снабжен набором элементарных паттернов поведения и базой знаний, с помощью которой он реализует так называемое реактивное поведение. Таким образом, моделируются функции лимбической системы, играющей роль среднего мозга чело- 


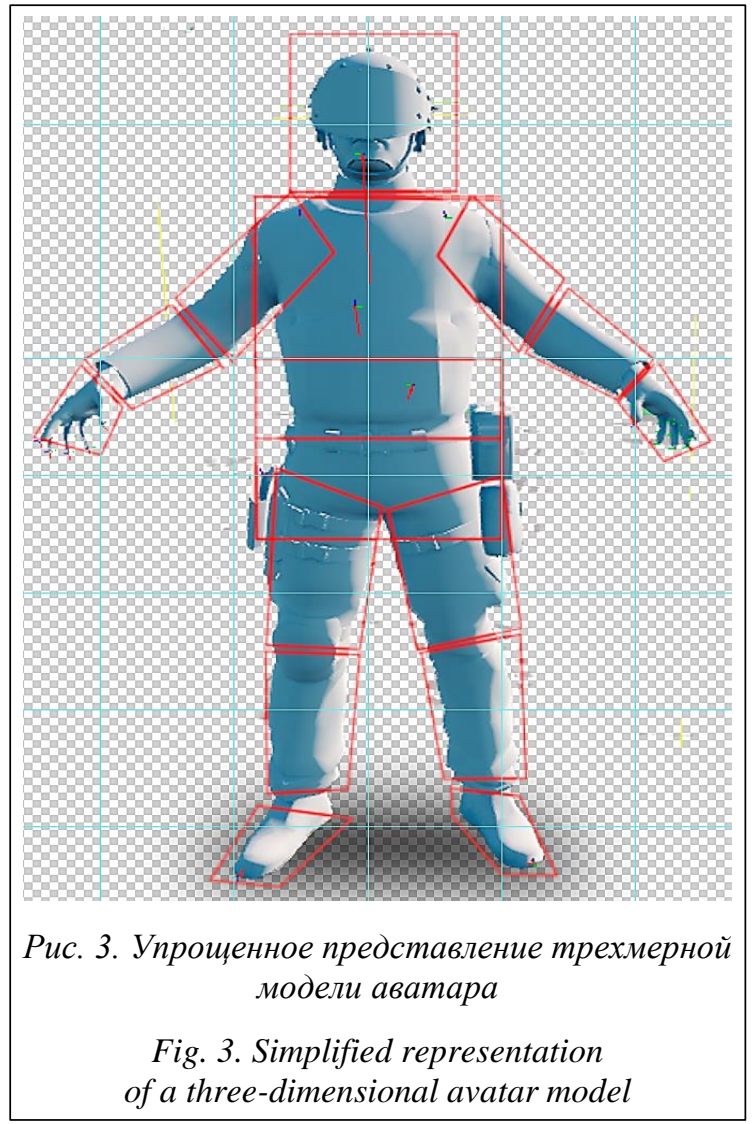

века. C ее помощью мозг обнаруживает так называемые значительные стимулы, угрожающие выживанию, и посылает информацию телу, чтобы оно могло реагировать на них надлежащим образом, минуя сознание и используя встроенные реакции. И только потом изменения в теле фиксируются сознанием, вызывая соответствующую эмоциональную оценку. Например, реакции страха или гнева начинаются в лимбической системе, что вызывает диффузное влияние на симпатическую нервную систему (она регулирует расход энергоресурсов и мобилизует организм в стрессовых ситуациях). Реакция организма, известная как «бей или беги», готовит человека к угрожающим ситуациям путем увеличения частоты его сердечных сокращений, дыхания или кровяного давления. В результате человек может в зависимости от обстоятельств защищаться или бежать. Аналогичным механизмом снабжен и аватар. Для этого он обеспечен зри- тельной функциональностью в виртуальной среде.

Реализация зрительной функции модели агента заключается в видении объектов через простые формы, например, в данном случае это кубы и их вершины, и игнорировании объектов, которые не представляют для модели ценности, например, стены и прочие. Глаз реализован как пустой объект, использующийся в качестве конечной точки для построения зрительного луча и находящийся на уровне головы, для реалистичности он анимирован для случаев поворота головы в процессе анимации персонажа.

Вся зрительная часть сводится к трем основным функциям:

- определение попадания объекта в поле зрения;

- определение дистанции до объекта;

- построение векторов от упрощенной модели объекта к объекту, отвечающему за глаза агента.

Эти функции служат для сортировки и, таким образом, ускорения процесса обработки объекта.

Функция обнаружения предмета в области видимости глаза применяется для того, чтобы рассматривать объекты, попавшие в зону видимости глаза, тем самым позволяя снизить затраты на подробную обработку всех объектов. Схематично реализация области видимости показана на рисунке 5. Синим цветом выделено местоположение агента в мире. Угол зрения составляет 120 градусов.

Обнаружение предмета на расстоянии видимости глаза - еще одна функция для сортировки объектов и экономии времени просчета, представляющая собой область, разбитую на приоритеты (рис. 5).

Зеленая зона - высокий приоритет, объекты в этой области будут выбираться всегда. Сейчас он равен 20 метрам. Также в этой зоне будет производиться именование объектов.

Желтая и красная зоны приоритетов будут выбраны в случае, если нет объектов в зеленой зоне. Сейчас эти зоны равны 50 и 100 метрам соответственно.

В дальнейшем функцию можно улучшить и по временным затратам: объекты, располо-

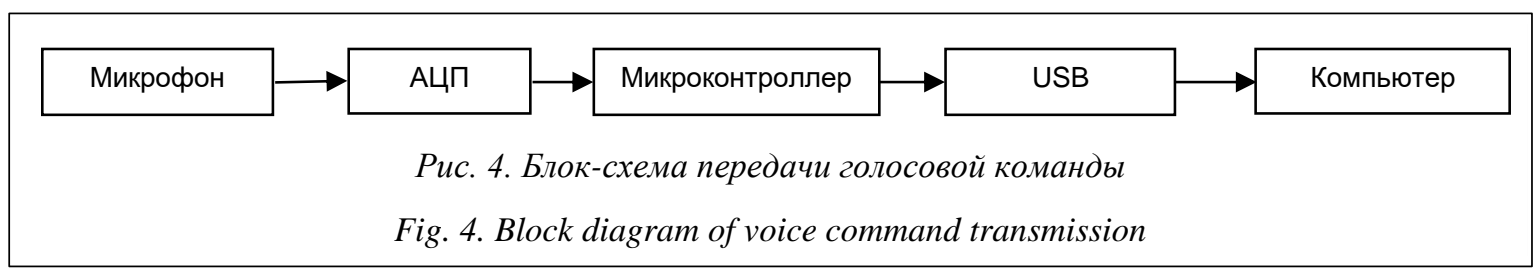




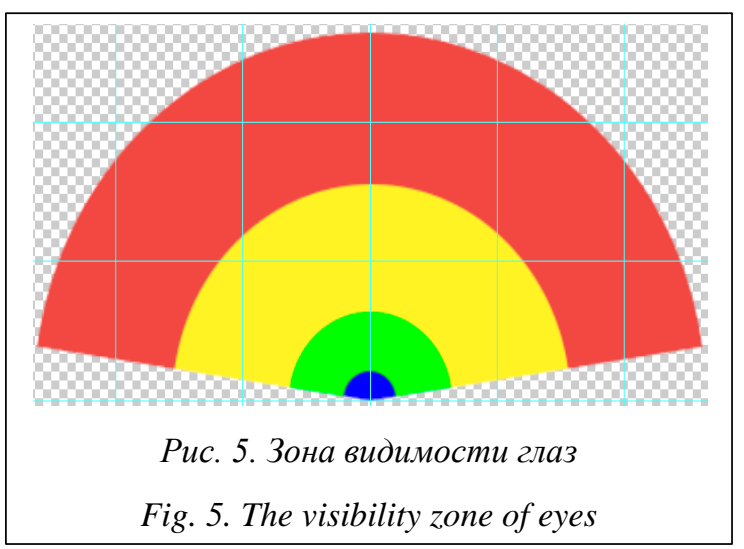

женные дальше от глаза, могут потребовать большего времени фокусировки.

Функция обнаружения пересечения между объектом и глазом работает по принципу нахождения пересечения между точками огрубленной модели объекта и глазом. Между двумя точками строится луч. Если на луч попадает какой-то объект, то эту точку глаз не видит. Если глаз видит хотя бы одну точку объекта, то объект видим полностью.

Испытуемый выполняет действия в соответствии со схемой, приведенной на рисунке 6.

В практической ситуации выбора при некотором множестве альтернатив $c \in C$ лицо, принимающее решение, выбирая конкретную альтернативу, руководствуется своими личными представлениями о лучших альтернативах. У разных лиц в одной и той же ситуации (при одном и том же $C$ ) представление о лучших альтернативах может различаться. При этом каждый из них может дать вполне рациональное объяснение сделанному выбору. Даже при выборе одних и тех же альтернатив разными лицами обоснования могут быть различными. Используя модель паттерна по наблюдаемому поведению в первой позиции, связанному с выбором в конкретной ситуации, можно сделать вывод о причинах, которые побудили сделать именно данный выбор, то есть восстановить логику выбора. Поэтому далее анализ полученного паттерна действия выполняется с точки зрения исследователя. Важно подчеркнуть, что для выполнения описанных агентом уже произведенных действий по собственному паттерну (рис. 6) исследователь должен выйти из своей позиции деятельности и перейти в новую позицию, внешнюю по отношению как к уже выполненным действиям, так и к будущей, проектируемой деятельности. Это называется рефлексией первого уровня: новая позиция агента, характеризуемая относительно прежней позиции, будет называться рефлексивной позицией, а знания, вырабатываемые в ней, рефлексивными знаниями, поскольку они берутся относительно знаний, выработанных в первой позиции. Приведенная схема рефлексивного выхода будет служить первой абстрактной модельной характеристикой рефлексии в целом.

Вторая позиция предполагает, возможно, полную имитацию поведения агента, когда исследователь, используя модель, полученную в первой позиции, пытается думать и действовать максимально приближенно к мыслям и поступкам агента. Такой подход позволяет понять на интуитивном уровне существенные, но неосознанные аспекты мыслей и действий моделируемого агента и уточнить модель.

Моделирование с третьей позиции заключается в наблюдении за поведением моделируемого агента в качестве незаинтересованного наблюдателя. Предполагается построение модели способа действия с точки зрения конкретной научной дисциплины, связанной с предметной областью агента.

Четвертая позиция предполагает своего рода интуитивный синтез всех полученных представлений с целью получить модель с максимальными значениями показателей удельной ценности по результату и эффективности.

Этот подход предполагает использование имплицитной и эксплицитной информации. Дело в том, что возможна ситуация, в которой агент знает или понимает суть некоторой деятельности, однако не способен осуществлять ее (осознанная некомпетентность). И наоборот, агент способен хорошо выполнять некоторые действия, но не понимает, как это делается (неосознанная компетентность).

Владение навыком в совершенстве подразумевает способность как делать то, что знаешь, так и знать, что делаешь. Тем не менее, многие поведенческие и психологические элементы, обеспечивающие успешность действий агентов, остаются по большей части неосознанными и лишь интуитивно понятными для них самих. В результате они не могут напрямую описать механизмы, лежащие в основе какихлибо способностей. Более того, некоторые агенты умышленно избегают размышлений о том, что и как они делают, опасаясь, что это знание помешает интуитивным действиям. Поэтому целями моделирования являются также выявление и идентификация неосознанной компетентности и доведение ее до сознания для лучшего понимания, совершенствования и передачи навыка. 


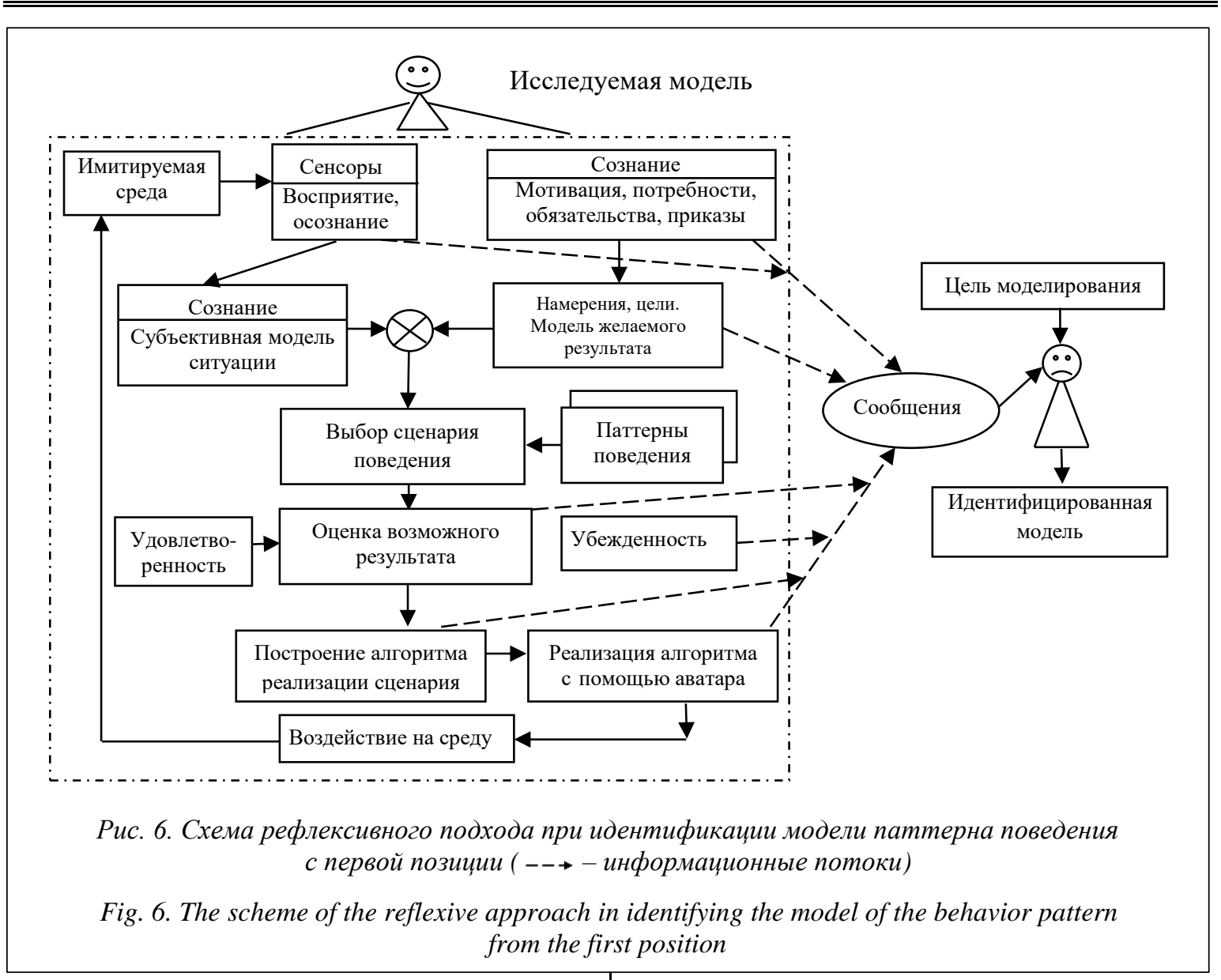

Когнитивная и поведенческая компетентность могут быть смоделированы либо имплицитно, либо эксплицитно. Имплицитное моделирование предполагает занятие второй позиции по отношению к субъекту моделирования для того, чтобы добиться интуитивного понимания субъективных переживаний данного человека. Эксплицитное моделирование состоит в переходе в третью позицию с целью описать явную структуру переживаний моделируемого агента таким образом, чтобы ее можно было передать другим. Имплицитное моделирование является, в первую очередь, индуктивным процессом, с помощью которого мы принимаем и воспринимаем структуры окружающего мира. По существу эксплицитное моделирование - дедуктивный процесс, с помощью которого мы описываем и осуществляем это восприятие. Оба процесса необходимы для успешного моделирования. Без имплицитной стадии не может быть эффективной интуитивной базы, на основе которой можно построить эксплицитную модель. С другой стороны, без эксплицитной фазы смоделированная информация не сможет воплотиться в приемах или средствах и быть переданной другим. Импли- цитное моделирование само по себе помогает человеку развивать личное, неосознанное умение в связи с желаемым поведением (так обычно учатся маленькие дети). Однако создание техники, механизма или навыка, которому можно научить или который можно передать другим, в каком-то смысле требует применения эксплицитного моделирования.

Результатом должна быть модель, в которой синтезированы интуитивное понимание способностей агента, непосредственные наблюдения за работой агента и эксплицитные познания исследователя в предметной области агента.

Экспериментальные исследования проведены для сравнительно простых поведенческих и когнитивных моделей паттернов, например, управление автономным подводным аппаратом, оценка боеготовности сил специального реагирования и ряда других. В результате реализации предлагаемых процедур были получены модели, в которых синтезированы интуитивное понимание способностей агента, непосредственные наблюдения за работой агента и эксплицитные познания исследователя в предметной области агента. 


\section{Заключение}

Интеллектуальные технологии, использующие теорию паттернов, имеют значительные перспективы, так как позволяют снять проблемы вычислительной сложности. Формальная модель паттерна поведения для системы управления автономным объектом, представленная в статье, описывает механизмы формирования субъективных представлений и оценок компонент ситуации выбора, модель выбора, учитывающую мотивы и обязательства. Показано, что построение модели предполагает выявление носителя наиболее успешной модели поведения (лидера).

Получение и анализ информации для идентификации модели основаны на четырех позициях ее восприятия и извлечения, получение информации - на активном эксперименте.
Показано, что записи команд с микрофона могут использоваться как временные ряды, содержащие реакцию оператора на моделируемые ситуации при выполнении миссии, то есть повторяющиеся паттерны в этих рядах представляют собой фиксацию паттернов поведения оператора. В результате идентификация паттерна поведения оператора осуществляется на основе выявления паттернов во временных рядах голосовых команд управления аниматом.

Обработка результатов предполагает синтез интуитивного понимания способностей агента, непосредственных наблюдений за работой агента, эксплицитных познаний исследователя в предметной области агента. Описанный подход использован при проектировании системы управления группой автономных необитаемых подводных аппаратов для выполнения поисковых и спасательных миссий.

Исследование выполнено при финансовой поддержке РФФИ, проект № 17-01-00728.

\section{Лuтература}

1. Nagatani K., Okada Y., Tokunaga N., Yoshida K., Kiribayashi S., Ohno K., Takeuchi E. et al. Multirobot exploration for search and rescue missions: a report of map building in RoboCupRescue 2009. Journal of Field Robotics, 2011, vol. 28, no. 3, pp. 373-387. DOI: 10.1002/rob.20389.

2. Городецкий В.И. Поведенческие модели киберфизических систем и групповое поведение: основные понятия // Изв. ЮФУ. Технические науки. 2019. № 1. С. 144-162.

3. Виноградов Г.П., Прохоров А.А. Ontologies in the problems of building domain model // Программные продукты и системы. 2018. № 4. С. 677-684. DOI: 10.15827/0236-235x.124.677-683.

4. Burrafato P., Cossentino M. Designing a multi-agent solution for a bookstore with PASSI methodology. Proc. of Intern. Conf. on AOIS, 2002, pp. 119-135.

5. Caire G., Coulier W., Garijo F., Gómez-Sanz J.J., Pavón J., Leal F. Agent oriented analysis using message/UML. Agent-Oriented Software Engineering II, 2002, vol. 2222, pp. 119-125. DOI: 10.1007/3-54070657-7_8.

6. Cohen P., Levesque H. Teamwork. Nous, 1991, vol. 25, pp. 487-515. DOI: 10.2307/2216075.

7. Cougaar Agent Architecture. URL: http://cougaar.org/wp/ documentation/tutorials/ (дата обращения: 19.11.2020).

8. DeLoach S. Analysis and design using MaSE and agentTool. Proc. XII MAICS, 2001. DOI: 10.21236/ ada448191.

9. Федунов Б.Е. Конструктивная семантика для разработки алгоритмов бортового интеллекта антропоцентрических объектов // Изв. РАН. ТиСУ. 1998. № 5. С. 796-796.

10. Виноградов Г.П. Моделирование принятия решений интеллектуальным агентом // Программные продукты и системы. 2010. № 3. С. 45-51.

11. Виноградов Г.П., Кузнецов В.Н. Моделирование поведения агента с учетом субъективных представлений о ситуации выбора // Искусственный интеллект и принятие решений. № 3. С. 58-72.

12. Deloach S., Garcia-Ojeda J. The O-MaSE methodology. In: Handbook on Agent-Oriented Design Processes, 2014, pp. 253-285. DOI: 10.21236/ada448191.

13. Filatova N., Bodrina N., Sidorov K., Shemaev P., Vinogradov G. Biotechnical system for the study of processes of increasing cognitive activity through emotional stimulation. Advances in Intelligent Systems and Computing, 2020, vol. 1156, pp. 548-558. DOI: 10.1007/978-3-030-50097-9_56.

14. Заде Л. Понятие лингвистической переменной и его применение к принятию приближенных решений. М.: Мир, 1976, 167 с.

15. Борисов П.А., Виноградов Г.П., Семенов Н.А. Интеграция нейросетевых алгоритмов, моделей нелинейной динамики и методов нечеткой логики в задачах прогнозирования // Изв. РАН. ТиСУ. 2008. № 1. С. $78-84$. 
16. Финн В.К. Далеко не все функции естественного интеллекта могут быть формализованы и автоматизированы // КоммерсантЪ. Наука. 2019. № 68. С. 33-34. URL: https://www.kommersant.ru/ doc/4198609 (дата обращения: 14.11.2020).

17. Bonomi F., Milito R., Zhu J., Addepalli S. Fog computing and its role in the internet of things. Proc. MCC, 2012, pp. 13-16. DOI: 10.1145/2342509.2342513.

18. Дилтс Р. Моделирование с помощью НЛП; [пер. с англ.]. Спб: Питер, 2010. 288 с.

19. Vinogradov G.P. A subjectve rational choice. Journal of Physics: Conf. Series, 2017, vol. 803, art. 012176. DOI: 10.1088/1742-6596/803/1/012176.

20. Городецкий В.И. Самоорганизация и многоагентные системы. І. Модели многоагентной самоорганизации // Изв. РАН. ТиСУ. 2012. № 2. С. 92-120.

21. Городецкий В.И. Самоорганизация и многоагентные системы. II. Приложения и технология разработки // Изв. РАН. ТиСУ. 2012. № 3. С. 102-123.

22. Foundation for Intelligent Physical Agents: FIPA ACL Message Structure Specification. URL: http://fipa.org/specs/fipa00061/SC00061G.pdf (дата обращения: 19.09.2020).

23. AgentBuilder - an integrated software toolkit that allows software developers to quickly develop intelligent software agents and agent-based applications. URL: http://www.agentbuider.com (дата обращения: 19.01.2016)

24. Bernon C., Gleizes M.-P., Peyruqueou S., Picard G. A methodology for adaptive multi-agent systems engineering. Proc. III ESAW, 2002, vol. 2577, pp. 156-169. DOI: 10.1007/3-540-39173-8_12.

\section{Approach to designing software for artificial entity management systems}

Vinogradov G.P. ${ }^{1,2}$, Dr.Sc. (Engineering), Professor, Head of Laboratory,wgp272ng@mail.ru

Konukhov I.A. ${ }^{2}$, Head of Department, konuhov@cps.tver.ru

Shepelev G.A. ${ }^{2}$, Engineer-Programmer

${ }^{1}$ Tver State Technical University, Tver, 170026, Russian Federation

${ }^{2}$ R\&D Institute Centerprogramsystem, Tver, 170024, Russian Federation

Abstract. The problems of intelligent control of artificial entities (including robotic complexes (RC)) are closely associated with the problem of decision-making. The formal decision theory was developed by abstracting from subjective factors. This led to the development of a normative theory of decision - making by the "ideal" subject. Analysis of approaches to the construction of RC control systems has shown that they do not have the property of independent decisionmaking. In practice, developers entertain possible behaviors of such systems, and the corresponding algorithms are embedded in the RC control system. As a result, such an object doesn't have a self-sufficient behavior that guarantees the fulfillment of some mission, especially as part of a human-machine system. The demand for behavior intellectualization forces us to reconsider the logical and mathematical abstractions underlying the construction of their onboard control systems.

The work objective is to substantiate the approach to software development of intelligent RC control systems based on the pattern theory. It is necessary to develop an approach that ensures the transfer of effective experience to the RC management system, the compatibility of the theological approach, and the causal approach, which is important when integrating the RC and the personnel of the units. Show that the patterns of the subject's departure from the ideal rational choice to the subjectively rational are associated with the peculiarities of identification and understanding of the state of the external environment and the properties of their interests. External factors are related to the obligations that the agent assumes. Internal factors reflect the interests of the subject, induced by his needs and the ethical system that he sticks to.

The paper uses the methods of the theory of reflexive games and the theory of information management of systems with will and intelligence.

It is shown that the choice in conditions of severe time deficit is made based on behavior patterns that reflect effective experience. Patterns form both the information structure of representations and the set of possible variants of representations. Assessments of contentment with the current situation of choice by the subject lead to a change in the structure of interests of the subject, and he can choose it. A formal model of the behavior pattern is developed. An approach to solving the problem of identification and construction of pattern models is proposed. For these purposes, four positions of information processing were used, and a method of logical inference on patterns was developed. The results of software solutions for identifying the behavior pattern when using a new generation of training systems are presented.

Keywords: decision-making, purposeful systems, fuzzy judgment, pattern, choice situation, RC.

Acknowledgements. The study was funded by RFBR according to the research project no. 17-01-00728. 


\section{References}

1. Nagatani K., Okada Y., Tokunaga N., Yoshida K., Kiribayashi S., Ohno K., Takeuchi E. et al. Multi-robot exploration for search and rescue missions: a report of map building in RoboCupRescue 2009. Journal of Field Robotics, 2011, vol. 28, no. 3, pp. 373-387. DOI: 10.1002/rob.20389.

2. Gorodetsky V.I. Behavioral model for cyber-physical system and group control: the basic concepts. Izv. SFedU. Engineering Sciences, 2019, no. 1, pp. 144-162 (in Russ.).

3. Vinogradov G.P., Prokhorov A.A. Ontologies in the problems of building domain model. Software and Systems, 2018, no. 4, pp. 677-684. DOI: 10.15827/0236-235x.124.677-683.

4. Burrafato P., Cossentino M. Designing a multi-agent solution for a bookstore with PASSI methodology. Proc. of Intern. Conf. on AOIS, 2002, pp. 119-135.

5. Caire G., Coulier W., Garijo F., Gómez-Sanz J.J., Pavón J., Leal F. Agent oriented analysis using message/UML. Agent-Oriented Software Engineering II, 2002, vol. 2222, pp. 119-125. DOI: 10.1007/3-540-70657-7_8.

6. Cohen P., Levesque H. Teamwork. Nous, 1991, vol. 25, pp. 487-515. DOI: 10.2307/2216075.

7. Cougaar Agent Architecture. Available at: http://www.cougaar.world/wp/documentation/tutorials/ (accessed November 19, 2020).

8. DeLoach S. Analysis and design using MaSE and agentTool. Proc. XII MAICS, 2001. DOI: 10.21236/ada448191.

9. Fedunov B.E. Constructive semantics of anthropocentric systems for development and analysis of specifications for onboard intelligent systems. Izv. RAN TiSU, 1998, no. 5, pp. 796-796 (in Russ.).

10. Vinogradov G.P. Model of decision-making by the intellectual agent. Software and Systems, 2010, no. 3, pp. 45-51 (in Russ.).

11. Vinogradov G.P., Kuzneczov V.N. Modeling agent's behavior based subjective perceptions on the situation of choice. Artificial Intelligence and Decision Making, 2011, no. 3, pp. 58-72 (in Russ.).

12. Deloach S., Garcia-Ojeda J. The O-MaSE methodology. In: Handbook on Agent-Oriented Design Processes, 2014, pp. 253-285. DOI: 10.21236/ada448191.

13. Filatova N., Bodrina N., Sidorov K., Shemaev P., Vinogradov G. Biotechnical system for the study of processes of increasing cognitive activity through emotional stimulation. Advances in Intelligent Systems and Computing, 2020, vol. 1156, pp. 548-558. DOI: 10.1007/978-3-030-50097-9_56.

14. Zadeh L.A. The concept of a linguistic variable and its application to approximate reasoning. Information Sciences, 1975, no. 8, pp. 199-249.

15. Borisov P.A., Vinogradov G.P., Semenov N.A. Integration of neural network algorithms, nonlinear dynamics models, and fuzzy logic methods in prediction problems. Izv. RAN TiSU, 2008, no. 1, pp. 78-84 (in Russ.)

16. Finn V.K. Not all natural intelligence functions can be formalized and automated. Kommersant. Nauka, 2019, no. 68, pp. 33-34. Available at: https://www.kommersant.ru/doc/4198609 (accessed November 14, 2021) (in Russ.).

17. Bonomi F., Milito R., Zhu J., Addepalli S. Fog computing and its role in the internet of things. Proc. MCC, 2012, pp. 13-16. DOI: 10.1145/2342509.2342513.

18. Dilts R. Modeling with NLP. 1998, 306 p. (Russ. ed.: St. Petersburg, 2010, 288 p.).

19. Vinogradov G.P. A Subjectve Rational Choice. Journal of Physics: Conf. Series, 2017, vol. 803, art. 012176. DOI: 10.1088/1742-6596/803/1/012176.

20. Gorodeczkij V.I. Self-organization and multiagent systems: I. Models of multiagent self-organization. Journal of Computer and Systems Sciences International, 2012, vol. 51, no. 2, pp. 256-281.

21. Gorodeczkij V.I. Self-organization and multiagent systems: II. Applications and the development technology. Journal of Computer and Systems Sciences International, 2012, vol. 51, no. 3, pp. 391-409.

22. Foundation for Intelligent Physical Agents: FIPA ACL Message Structure Specification. Available at: http://fipa.org/specs/fipa00061/SC00061G.pdf (accessed September 09, 2020).

23. AgentBuilder - an Integrated Software Toolkit that Allows Soft-Ware Developers to Quickly Develop Intelligent Software Agents and Agent-Based Applications. Available at: http://www.agentbuilder.com (accessed January 19, 2016).

24. Bernon C., Gleizes M.-P., Peyruqueou S., Picard G. A methodology for adaptive multi-agent systems engineering. Proc. III ESAW, 2002, vol. 2577, pp. 156-169. DOI: 10.1007/3-540-39173-8_12.

\section{Для цитирования}

Виноградов Г.П., Конюхов И.А., Шепелев Г.А. Подход к проектированию программного обеспечения систем управления искусственными сущностями // Программные продукты и системы. 2021. T. 34. № 1. C. 005-018. DOI: 10.15827/0236-235X.133.005-018.

\section{For citation}

Vinogradov G.P., Konukhov I.A., Shepelev G.A. Approach to designing software for artificial entity management systems. Software \& Systems, 2021, vol. 34, no. 1, pp. 005-018 (in Russ.). DOI: 10.15827/0236-235X.133.005-018. 